\title{
Archives
}

\section{Le traitement et la diffusion du fonds Armand-Frappier (ou comment être opportuniste en huit leçons)}

FRANÇOIS CARTIER

Volume 47, numéro 2, 2018

URI : https://id.erudit.org/iderudit/1045165ar

DOI : https://doi.org/10.7202/1045165ar

Aller au sommaire du numéro

\section{Éditeur(s)}

Association des archivistes du Québec (AAQ)

ISSN

0044-9423 (imprimé)

2369-9256 (numérique)

Découvrir la revue

Citer cet article

CARTIER, F. (2018). Le traitement et la diffusion du fonds Armand-Frappier (ou comment être opportuniste en huit leçons). Archives, 47(2), 7-20.

https://doi.org/10.7202/1045165ar
Résumé de l'article

Cet article nous relate les différentes étapes qui ont mené à la diffusion du Fonds Armand-Frappier. Ce fonds, déposé aux archives de l'Institut national de la recherche scientifique (INRS, une constituante du réseau de l'Université du Québec), illustre le parcours de cet homme qui eut une influence importante sur l'évolution des sciences au Québec. C'est le $25^{\mathrm{e}}$ anniversaire du décès du Dr Frappier qui a fourni le prétexte à ce projet de diffusion qui a finalement compris une exposition virtuelle et un programme de conférence. Pour arriver à leurs fins, l'équipe du service des archives de l'INRS a fait preuve de créativité et d'opportunisme afin d'aller chercher ressources et financement. Le projet a demandé dans un premier temps un nouveau traitement du fonds et a fait surgir de nombreuses questions quant au passage d'un format de description à un autre ainsi que sur les outils technologiques à utiliser.

L'auteur aura retenu huit leçons au cours de ce projet. Il nous les livre ici par la description de sa mise en oeuvre. Ces leçons concernent, entre autres, la capacité de vendre notre idée, celle de saisir les opportunités et la nécessité de bien choisir ses collaborateurs et partenaires. Il revient également sur certains éléments de gestion, tels que l'évaluation du temps requis et l'utilité du plan B. Il nous incite finalement à réaliser la richesse de nos fonds et de l'intérêt qu'ils suscitent chez les jeunes. Cet article nous démontre qu'avec de l'initiative et la maximisation des programmes à notre disposition, il est possible de réaliser des projets qui deviennent des réussites.
Ce document est protégé par la loi sur le droit d'auteur. L'utilisation des services d'Érudit (y compris la reproduction) est assujettie à sa politique d'utilisation que vous pouvez consulter en ligne.

https://apropos.erudit.org/fr/usagers/politique-dutilisation/ 


\section{BILAN D'EXPÉRIENCE}

\section{Le traitement et la diffusion du fonds Armand-Frappier (ou comment être opportuniste en huit leçons)}

\section{FRANÇOIS CARTIER}

Archiviste, Institut national de la recherche scientifique

En avril 2014, je me joins à l'équipe du Service des archives et de la gestion documentaire de l'Institut national de la recherche scientifique (INRS). J'y arrive avec un bagage d'archiviste ayant surtout œuvré dans des musées d'histoire. De vieux manuscrits, des daguerréotypes, j'en ai vu jusqu'à plus soif. L'INRS est une université de recherche fondée en 1969 qui fait partie du réseau de I'Université du Québec. Elle est constituée de quatre centres de recherche au Québec, chacun ayant ses propres domaines de recherche: un à Varennes (Énergie, Matériaux et Télécommunications), un à Montréal (Urbanisation, Culture et Société), un à Québec (Eau, Terre et Environnement) et un à Laval (Microbiologie, ex-Institut Armand-Frappier ${ }^{1}$ ). Me voilà donc dans un organisme public relativement jeune, avec une gouvernance et une culture à apprivoiser et où je dois rafraîchir mes notions de gestion documentaire. Sortir de sa zone de confort, même dans la quarantaine, ça peut être intimidant! Je dois toutefois avouer que je ressentais un certain plaisir à me reconnecter à mon premier amour, la science, même si j'avoue 
ne comprendre qu'à moitié la nature des recherches menées par les scientifiques de I'INRS.

Peut-être est-ce pourquoi, parallèlement à mon apprentissage de nouveau gestionnaire de documents en milieu universitaire, j'ai décidé de donner un peu d'attention aux histoires scientifiques cachées dans les archives historiques qui se trouvaient maintenant sous ma responsabilité. Pour diverses raisons, notamment la petitesse de l'équipe en place (deux archivistes et une technicienne en documentation), la majorité des efforts de notre service sont consacrés à gérer les documents issus des différentes unités de I'INRS. En effet, les besoins opérationnels gardent notre équipe occupée à temps plein. En conséquence, les archives historiques demeurent un peu laissées à elles-mêmes. Un seul groupe de documents, les archives personnelles du $D^{r}$ Armand Frappier, avait déjà fait l'objet d'un premier traitement en 1988. Ces documents ne demandaient qu'à s'ouvrir davantage aux chercheurs.

\section{UN PROJET DE TRAITEMENT PREND FORME}

C'est donc sur ce fonds que j'ai décidé de me concentrer. L'histoire de ces documents est assez particulière et commence bien avant la fondation de I'INRS. Armand Frappier (1904-1991) est devenu l'un des scientifiques les plus célèbres au Canada dans la seconde moitié du XXe siècle. II a jeté les bases de la microbiologie moderne au Québec et a été responsable de grandes campagnes de vaccination, notamment contre la tuberculose, une maladie qui faisait des ravages dans la population².

Ses archives couvrent donc sa vie personnelle, mais surtout sa carrière de scientifique et de directeur de l'institut qui en viendra à prendre son nom. C'est d'ailleurs en 1999 que l'Institut Armand-Frappier ${ }^{3}$ est intégré à I'INRS. Voilà donc pourquoi nous avons aujourd'hui les archives du célèbre scientifique.

En 1988, le fonds a fait l'objet d'un traitement par l'équipe des archives de l'Institut Armand-Frappier. L'archiviste alors en place, Maryse Odesse, a bénéficié d'un luxe que de nombreux archivistes n'ont pas: la présence et les conseils du créateur des archives. En effet, le $D^{r}$ Frappier, même à son âge avancé (il avait alors 84 ans), avait lui-même supervisé le traitement de ses archives. Ceci coïncidait d'ailleurs avec son propre projet, qui consistait à rédiger ses mémoires ${ }^{4}$. 


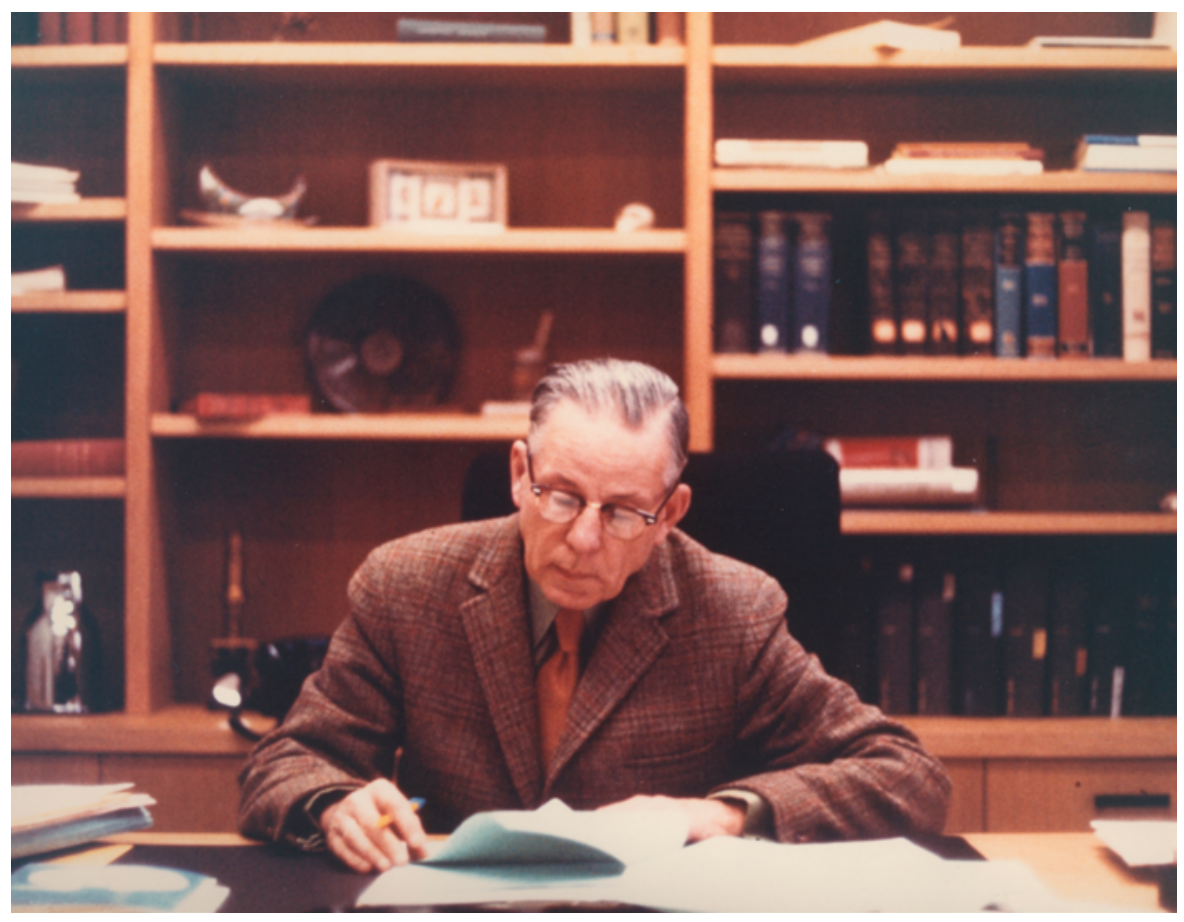

Figure 1: Armand Frappier dans son bureau, dans les années 1970.

Fonds de I'Institut Armand-Frappier. Photographe: André Levac.

Un inventaire détaillé de ses archives est issu de ce projet et a contribué à donner un premier aperçu du contenu du fonds. Ces archives serviront entre autres à la production d'un documentaire biographique sur le $D^{r}$ Frappier réalisé en 1995 par Nicole Gravel ${ }^{5}$. Par contre, même avec un inventaire détaillé à la clé, le fonds demeure sous-exploité. II faut noter que même si l'instrument de recherche était remarquablement détaillé, une absence d'indexation rendait son utilisation laborieuse. Sa publication survient aussi avant l'introduction des Règles pour la description des documents d'archives (RDDA) (en 1990), ce qui le rend non conforme au format de description encore en vigueur de nos jours. Toutefois, au-delà de l'importance de son créateur et de son accessibilité limitée, le nouveau traitement de ce fonds a été rendu nécessaire par la présence d'une vingtaine de boîtes de documents supplémentaires qui n'avaient jamais été intégrées au fonds. II s'agissait de documents que le $D^{r}$ Frappier avait encore en sa possession lors de son décès et qui avaient tout bonnement été rangés sur des tablettes d'un de nos dépôts. 


\section{MISER SUR LES PROGRAMMES DE SOUTIEN}

Mon projet était donc relativement simple: intégrer ces documents au fonds existant et traiter de nouveau ce dernier au grand complet. Comme le personnel du Service des archives et de la gestion documentaire (SAGD) était déjà fort occupé, une ressource additionnelle était requise pour débuter ce projet. Peu après mon arrivée à I'INRS, j'avais déjà commencé à accueillir des stagiaires au sein de notre équipe. Toutefois, traiter un tel fonds demandait une intervention plus soutenue que vingt jours ouvrables.

J'ai donc fait appel au programme de Soutien au traitement des archives de Bibliothèque et Archives nationales du Québec (BAnQ). Je connaissais bien ce programme pour y avoir fait appel plusieurs fois dans le passé. Je me disais qu'avec un fonds issu d'un personnage comme Armand Frappier, mes chances seraient bonnes.

- Leçon numéro un : bien vendre notre projet. II ne suffit pas d'y croire ; il faut que les autres y croient aussi.

La réponse de BAnQ a été positive. J'ai donc été en mesure d'embaucher une personne contractuelle en mai 2015 pour commencer à traiter le fonds. Même si son mandat n'était que de deux courts mois, c'était déjà assez pour abattre pas mal de besogne. Le travail principal a consisté à prendre connaissance du contenu des «nouvelles» boîtes du fonds et de mettre sur pied un plan de classification dans lequel se fonderaient les « deux fonds » Armand-Frappier. Comme il arrive souvent, les principaux défis ont été le volume des documents et le peu de temps pour tout traiter. En conséquence, une fois les deux fonds intégrés, nous nous retrouvions devant un fonds d'une taille surprenante de près de 20 mètres linéaires. À la place d'un plan de classification principalement basé sur les types de documents, nous avions maintenant un plan façonné selon les activités personnelles et professionnelles d'Armand Frappier. Avec l'aide de stagiaires accueillis au cours de l'été 2015, le plan de classification a pu être raffiné et le classement physique des documents fut complété. Les descriptions des niveaux supérieurs (fonds et séries) ont aussi été rédigées.

- Leçon numéro deux: c'est toujours plus long que prévu. On a beau ajouter des heures supplémentaires au projet pour couvrir les contingences, le temps finira toujours par manquer. 


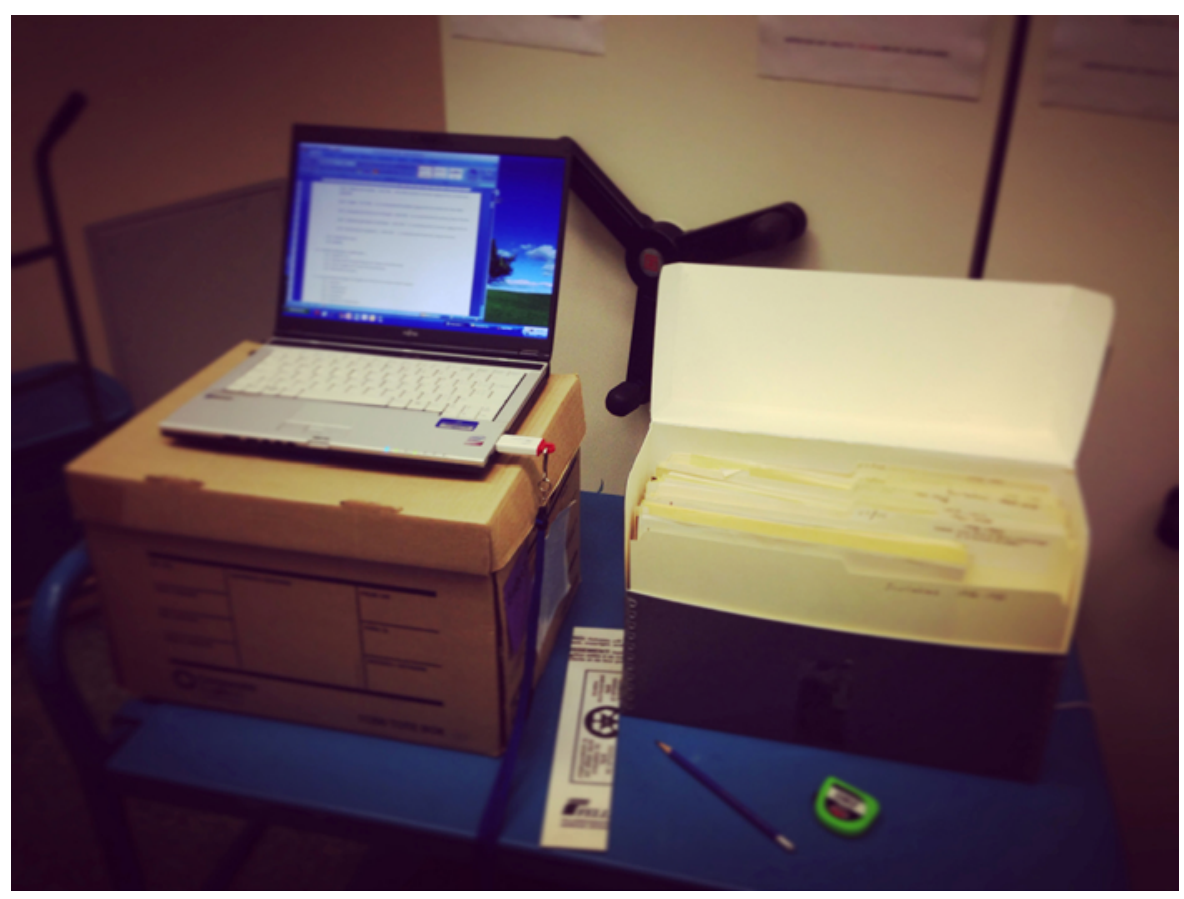

Figure 2: Le fonds Armand-Frappier en voie d'être traité. Photographe: François Cartier.

\section{CHOISIR DES OUTILS TECHNOLOGIQUES ADAPTÉS À SES MOYENS ET SES BESOINS}

À mon arrivée à I'INRS, aucune base de données n'est utilisée pour gérer les documents historiques. Nous étions donc devant un choix: soit laisser nos notices dans un logiciel comme Word, soit procéder à l'acquisition d'un système informatique permettant d'y insérer nos notices. Comme nous étions alors en plein magasinage pour un logiciel de gestion intégrée des documents, il était hors de question d'utiliser nos ressources financières déjà limitées pour acheter une application qui servirait à décrire et à indexer nos archives historiques. Ce travail, je l'espérais, pourrait être fait grâce à notre futur logiciel de GID .

J'ai donc opté pour le logiciel AtoM un outil développé sous l'égide du Conseil international des archives (ICA) en collaboration avec plusieurs partenaires partout dans le monde, comme Bibliothèque et Archives 
Canada ${ }^{7}$. Bien que le logiciel ait été développé sur mesure pour les normes de description développées par I'ICA (ISAD-G, ISAAR-CPF, ISDF et ISDIAH ${ }^{8}$ ), une interface a été créée afin d'y consigner des notices conformes aux RDDA. De plus, AtoM est créé dès son origine comme un logiciel libre afin de pouvoir être adopté sans frais par les institutions d'archives. Plusieurs organismes l'ont déjà adopté, dont les Archives de la Ville de Montréal et le portail Archives Canada. AtoM servirait donc dans l'immédiat comme base de données pour les archives historiques de I'INRS. Vu que les infrastructures informatiques étaient disponibles, nous avons installé le programme sur nos propres serveurs ${ }^{9}$. Une décision sera prise éventuellement quant à la poursuite - ou non de son utilisation.

\section{4. ÊTRE STRATÉGIQUE DANS LA FAÇON DE METTRE EN VALEUR SON PROJET}

J'avais donc un fonds classé, étiqueté, mis en boîte et hébergé dans un système dédié, mais partiellement décrit. Heureusement, je savais déjà comment poursuivre mon projet. Un projet de traitement ou de diffusion (ou les deux) ne doit pas être mené "dans le vide», sans être relié à autre chose. Pour tirer profit des efforts investis dans de tels projets, il est toujours souhaitable de rattacher son projet à un autre élément. C'est un peu comme attacher son wagon à une locomotive en marche: on amène son propre bagage à un convoi déjà en mouvement.

Dans mon cas, c'était doublement facile. Premièrement, le $D^{r}$ Frappier est décédé en 1991. Donc, en 2015, nous étions à la veille de célébrer le $25^{\mathrm{e}}$ anniversaire de son décès. Ensuite, en regardant dans les sources de financement disponibles, j'ai vu que Patrimoine canadien offrait des subventions dans le cadre de programmes visant à commémorer les guerres mondiales ${ }^{10}$. Or, le Dr Frappier et ses collaborateurs, à partir de 1943, ont contribué à l'effort de guerre du Canada en fournissant des produits biologiques pour l'armée canadienne (notamment du sang desséché pour soigner les blessés). 


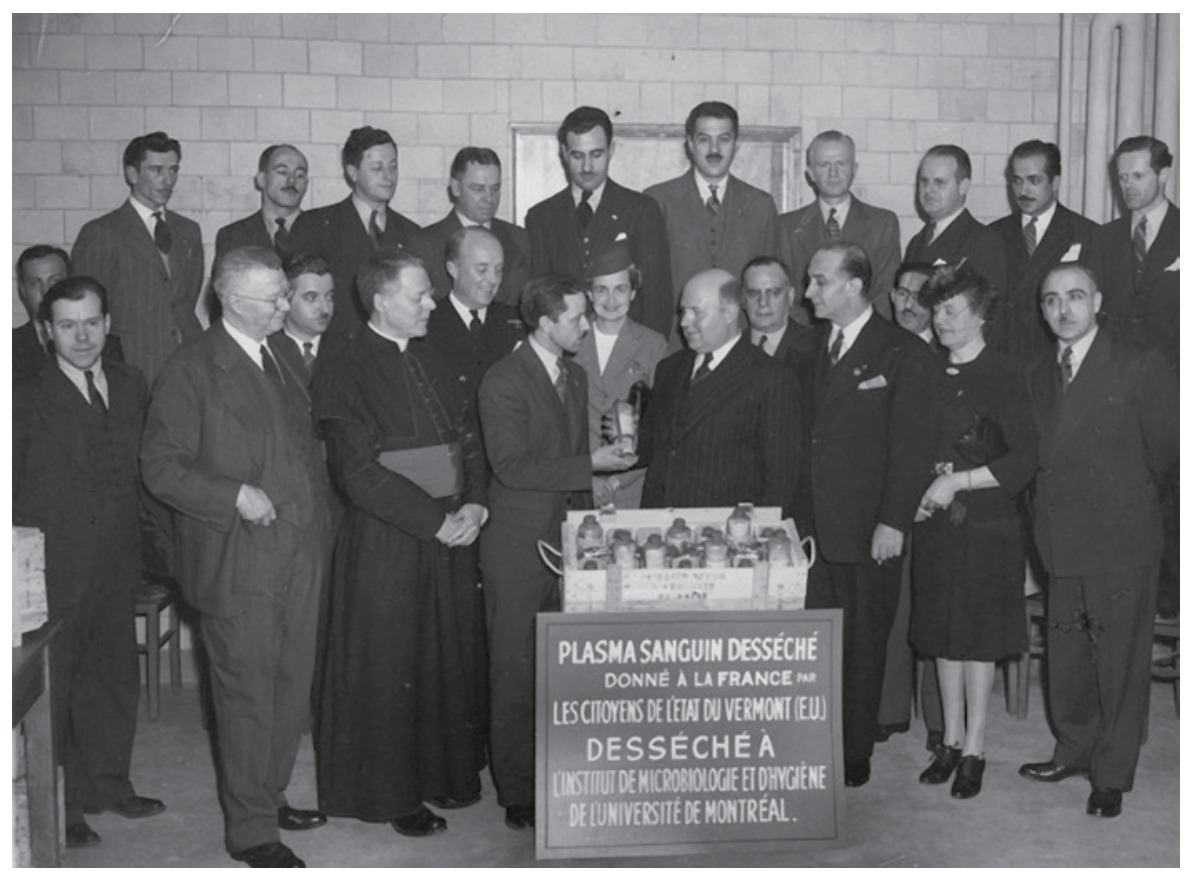

Figure 3: Don de sang desséché par l'équipe du D' Frappier en 1944 pour le bénéfice des citoyens français éprouvés par la guerre. Fonds de l'Institut Armand-Frappier.

Un projet d'exposition virtuelle a donc été soumis afin de souligner la contribution du $D^{r}$ Frappier et de son équipe à l'effort de guerre du Canada pendant la Seconde Guerre mondiale. Comme il s'agissait de la prolongation du travail effectué grâce à la subvention de BAnQ, je n'ai pas hésité à inclure BAnQ (par le biais de sa subvention) comme un " partenaire» dans notre projet de mise en valeur des archives d'Armand Frappier. Le programme de financement de Patrimoine canadien favorisait en effet la création de partenariats. Le financement obtenu de BAnQ a donc été intégré dans le budget de notre projet comme un apport d'un collaborateur externe. Mon simple projet de traitement commençait donc à se déployer comme une initiative de plus grande envergure. De plus, je prévoyais utiliser certaines pièces du Musée Armand-Frappier dans I'exposition virtuelle que nous allions mettre en ligne ${ }^{11}$. J'ai donc approché le musée et sa directrice afin d'obtenir un soutien additionnel. Une fois cet appui obtenu, j'avais un très bon dossier à présenter à Patrimoine canadien. Avec tous ces atouts en main, il n'est pas surprenant que nous ayons obtenu le financement demandé. 
- Leçon numéro trois: il faut savoir bien profiter des avantages qui sont mis à notre disposition.

Grâce à ce financement, à un peu d'aide de stagiaires et à la participation du personnel de mon équipe (moi inclus), nous avons pu terminer le traitement du fonds Armand-Frappier, notamment par une classification et une description plus fines des niveaux inférieurs du fonds. Une partie des sommes a aussi servi à monter une station de numérisation en vue de la mise en ligne de documents dans l'exposition virtuelle. L'avantage, ici, est qu'un numériseur et un ordinateur neufs sont venus s'ajouter à notre équipement permanent.

Les sommes accordées m'ont aussi permis de partir à la chasse pour trouver quelqu'un pour faire les recherches dans les documents, en sélectionner en vue de la numérisation et écrire des textes pour donner du contenu à l'exposition virtuelle. II me fallait donc trouver cette perle rare, qui avait à la fois des qualités en analyse, en recherche, en rédaction et qui savait comment bien numériser des documents. De plus, comme la subvention provenait du gouvernement fédéral, je devais mettre en ligne des contenus en français et en anglais. Je pouvais faire une première traduction des textes moi-même, mais je ne suis pas un traducteur professionnel.

Fort heureusement, j'ai trouvé cette perle rare. Une jeune diplômée du certificat en archivistique de l'École de bibliothéconomie et des sciences de l'information à qui j'avais déjà enseigné, et qui avait aussi fait des études en histoire et en anglais.

- Leçon numéro quatre: choisissez bien vos collaborateurs, car lorsque vous choisissez les mauvaises personnes, vous serez souvent déçus; mais si vous choisissez bien et que vous tablez sur leurs forces, vous en serez grandement récompensés.

À partir du début de 2016, environ quatre mois ont donc été consacrés à la recherche, à la numérisation, à la rédaction, à la révision et à la traduction. C'était là la réelle période intense du projet, où les textes sont écrits, lus et relus, corrigés, raccourcis et retravaillés. Ce fut aussi à cette étape que nous avons eu la difficile tâche de choisir les documents à numériser. Bien entendu, nous avions une grande quantité de photographies à notre disposition, mais nous disposions aussi d'une quantité importante de documents textuels à fort potentiel informatif et évocateur. C'est ici qu'il a fallu apprendre à bien choisir et à se montrer parcimonieux: mieux vaut 
choisir quelques excellents documents qui collent bien à nos textes qu'une marée de documents dans laquelle se dilue le propos. J'ai appris avec le temps qu'un des meilleurs critères est de choisir des images montrant des gens. Des édifices, c'est bien beau, mais les personnages sont généralement beaucoup plus évocateurs. De plus, les textes qui font parler les gens sont les meilleurs, d'où le choix de la correspondance.

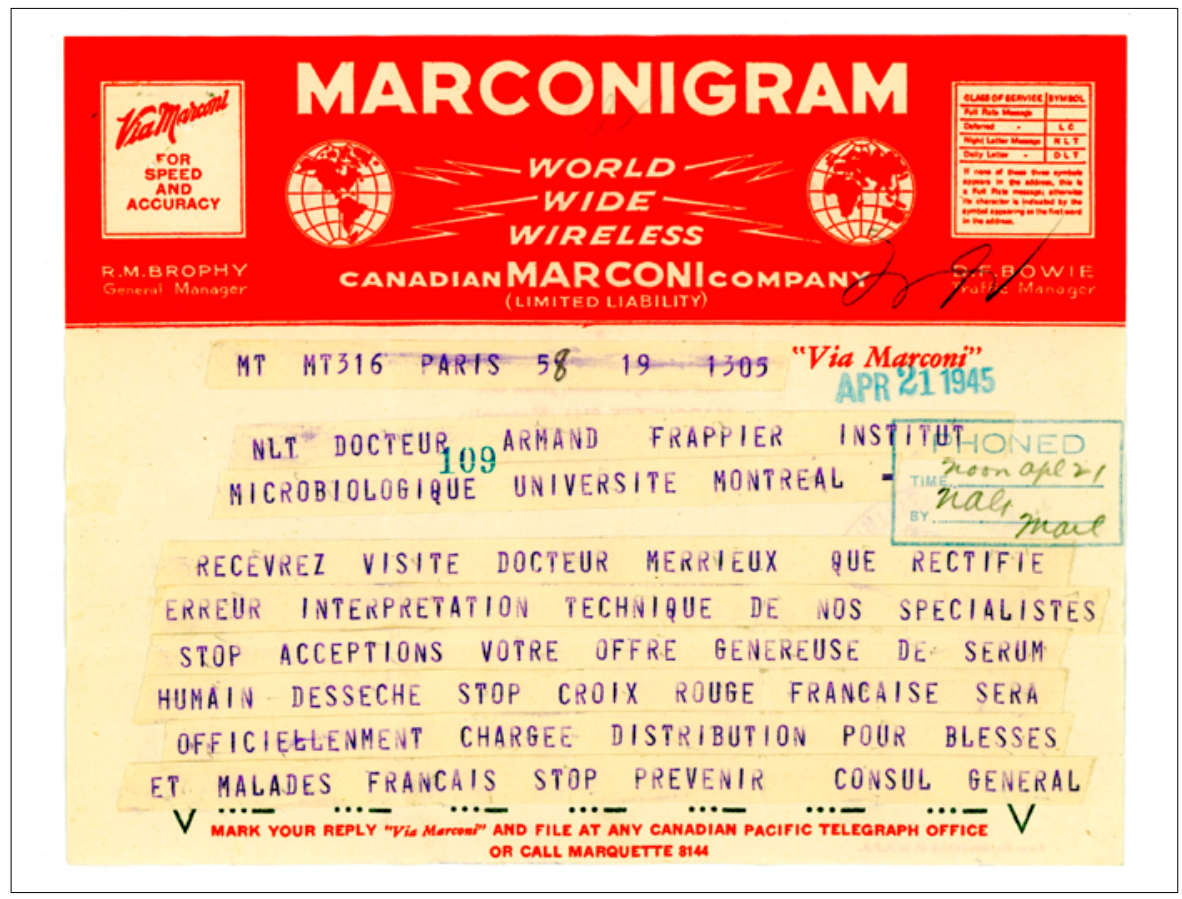

Figure 4: Exemple d'un document qui a été utilisé dans le cadre de notre exposition virtuelle. Fonds Armand-Frappier.

C'est surtout à ce moment qu'un plan de travail est testé: les délais accordés à chaque étape étaient-ils réalistes? Les impondérables, bien anticipés? Finalement, seuls quelques textes ont «débordé » au-delà de notre plan initial et ont dû être complétés après le départ de notre ressource contractuelle. Pas si mal, en fin de compte.

II restait alors à mettre en place les deux derniers morceaux de notre casse-tête: I'intégration de nos contenus sur le Web, de même que les activités de promotion, afin de faire connaître notre produit. 
Nous avions initialement prévu diffuser notre exposition virtuelle sur le site Web du Musée Armand-Frappier. En effet, le musée y présente déjà plusieurs artéfacts en lien avec la carrière du Dr Frappier. Toutefois, les responsables du musée m'ont informé en cours de projet qu'ils avaient décidé d'effectuer une refonte majeure de leur site Web. Notre exposition virtuelle, dans ce contexte, ne pourrait trouver sa place en ligne avant l'échéance du projet (et avant les délais fixés par Patrimoine canadien pour notre propre projet).

- Leçon numéro cinq : lors d'un projet mené en partenariat, s'assurer de bien s'entendre sur les priorités de chacun.

et

- Leçon numéro six: toujours avoir un «plan B».

Fort heureusement, nous avions ce plan de rechange: notre propre site Web et mes très compétents collègues du Service des communications de I'INRS. Deux personnes de cette équipe étaient donc prêtes à faire une dernière relecture des textes et à mettre images et textes en ligne dans une interface sobre qui s'harmonisait bien avec le look du site Web de l'INRS.

La science au service des blessés L'effort de guerre d'Armand Frappier Bienvenue dans notre exposition virtuelle sur la contribution du D' Armand Frappier et de
I'Institut de microbiologie et d'hygiène de l'Université de Montréal à l'effort de guerre du Canada
pendant la Seconde Guerre mondiale.

Nous vous invitons a decouvrir une facette meconnue drune histoire qui voit les scientifiques canadiens se mobiliser pour soutenit les soldats envoyts combattre outre-mer. Plus specifiquement, nous présentons les efforts deployes par Armand Frappier et son jeune institut pour produire et envoyer au tront du sang desseche pour les blesses.

Plus qưune cuuve de guerre, il s'agit d'une initiative qui permetra a rinsttut de croltve et de le positionner comme une des principales institutions de sante publique au Quebec et au Canada

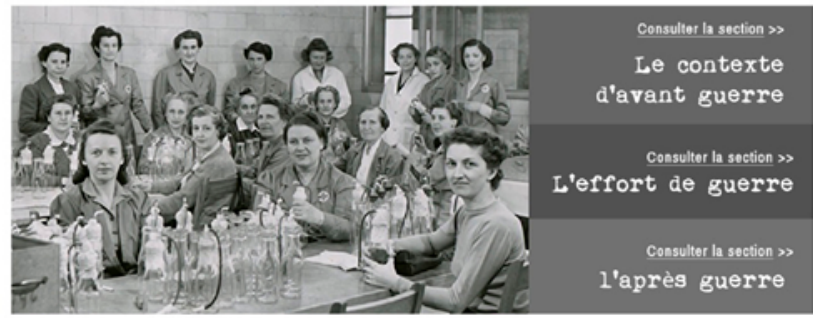

Le contexte đavant guerre >>

Figure 5: Sobre mais efficace: la page d'accueil de notre exposition virtuelle. 
Le produit final, bien que mettant l'accent sur la contribution de l'équipe du Dr Frappier à l'effort de guerre du Canada, servait aussi à illustrer les débuts de sa carrière, de son Institut et du rôle qu'il a joué dans la modernisation de la microbiologie au Québec. En conclusion, nous rendions bien honneur à ce grand scientifique, un quart de siècle après son décès.

\section{RENTABILISER SES EFFORTS ET EXPLOITER LE RÉSULTAT DE SON TRAVAIL DE DIFFÉRENTES FAÇONS}

En complément, une activité pédagogique est venue s'ajouter à l'exposition. Après tout, il s'agissait d'une exposition virtuelle présentée sur le site d'une université. De plus, pourquoi ne pas exploiter des contenus qui ont exigé beaucoup de travail de notre part?

- Leçon numéro sept: rentabilisez vos efforts et exploitez le résultat de votre travail de différentes façons.

Sans nécessairement le mentionner explicitement, j'avais choisi d'exploiter la filière de l'épistémologie, ou l'étude critique de la science. En effet, étant donné que l'institut du $D^{r}$ Frappier connut un essor réel pendant (et à cause de) la guerre, il devenait logique de susciter chez les élèves un questionnement relatif au rôle des sciences en temps de guerre, des guerres dans l'évolution de la science et des sociétés, de même qu'à la présence de la science dans la société en général.

À l'aide des textes de l'exposition virtuelle, de même que de deux textes d'historiens traitant de la question du progrès scientifique, l'idée était d'animer des discussions à partir de questions bien ciblées, comme "La fin justifie-t-elle les moyens?», ou "La guerre doit-elle être vue comme un mal nécessaire qui favorise l'avancement des sciences et technologies? », ou encore «Une société devrait-elle tolérer que la science soit exploitée en temps de guerre? Si oui, quelles balises éthiques ou morales devrait-on privilégier?».

Encore ici, l'important est un arrimage approprié: celui des thèmes de notre activité pédagogique au programme officiel du ministère de l'Éducation et de l'Enseignement supérieur (MEES). À cause du sujet, les élèves du second cycle du secondaire $\left(3^{\mathrm{e}}, 4^{\mathrm{e}}\right.$ et $5^{\mathrm{e}}$ secondaires) sont 
devenus notre clientèle cible, notamment dans le domaine d'apprentissage de l'univers social et des compétences de communication et d'exercice de son jugement.

- Leçon numéro huit: les jeunes sont prêts à apprendre. Vous disposez de matériel plein de potentiel. Allez les chercher avec vos histoires.

Toujours dans le but de faire fructifier le résultat de nos efforts, la dernière ligne droite du projet a justement eu pour objet de raconter I'histoire que nous avions mise en valeur. En plus de la promotion sur les réseaux sociaux et sur le site Web institutionnel de l'INRS, la méthode choisie a été de présenter deux conférences: une au Musée ArmandFrappier, I'autre à la Société d'histoire et de généalogie de Salaberry ${ }^{12}$ (Salaberry-de-Valleyfield étant le lieu de naissance du $D^{r}$ Frappier). Le partenariat avec le Musée Armand-Frappier a même permis quelques surprises, comme la présentation du violon original sur lequel a joué le $D^{r}$ Frappier, qui était excellent musicien.

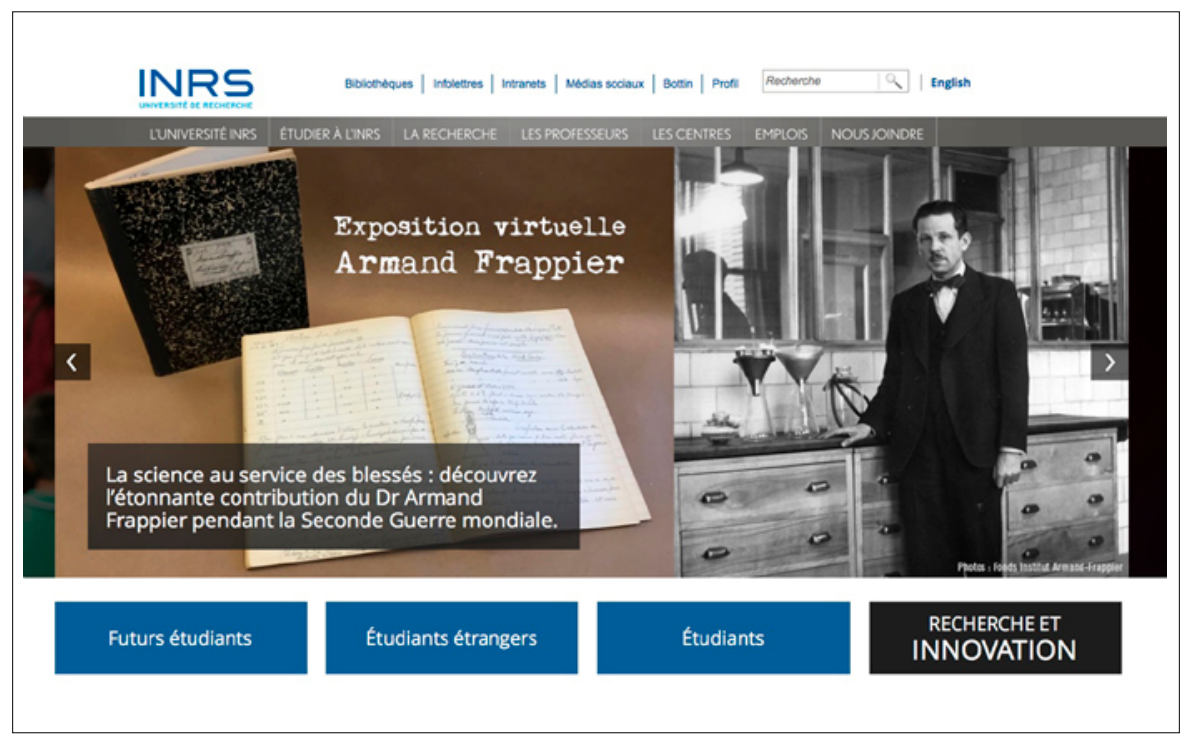

Figure 6: Bandeau promotionnel pour l'exposition virtuelle sur le site Web de I'INRS. 


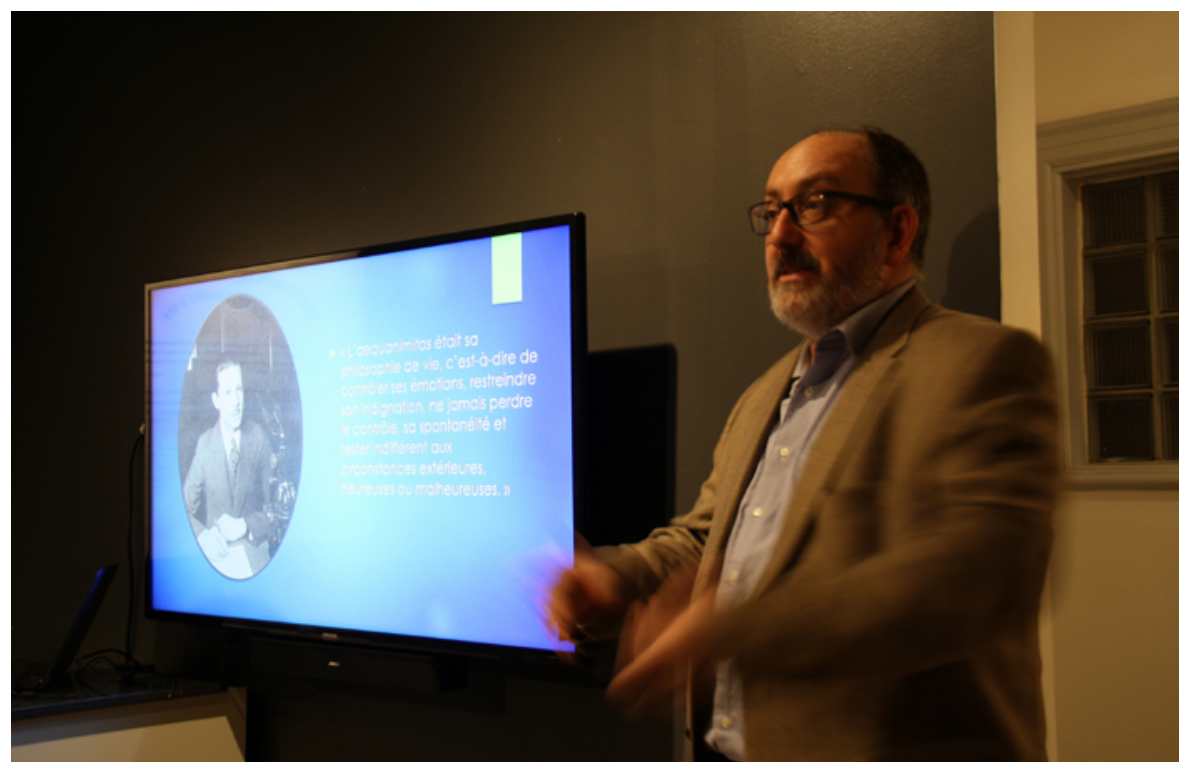

Figure 7: L'auteur pendant une des conférences sur le D' Frappier et son œuvre. Source: Musée Armand-Frappier.

Plus de 110 personnes ont ainsi été rejointes dans le cadre de ces conférences. De plus, le taux de fréquentation de notre exposition virtuelle donne déjà de bons résultats.

En résumé, avec moins de $28000 \$$, il a été possible de traiter un fonds de près de 20 mètres linéaires, de mettre en ligne une exposition simple mais très bien présentée, d'acheter du matériel informatique, de proposer aux élèves du second cycle du secondaire une activité de réflexion sur l'histoire des sciences, de présenter deux conférences et d'écrire le présent article. Les défis ont été nombreux, comme le nombre des documents à traiter. Cependant, en parachevant le projet grâce au soutien de ressources internes, comme nos collègues des communications, mais aussi avec l'aide de stagiaires toujours prêts à appliquer leurs connaissances, nous avons su démontrer qu'avec un peu de créativité, il est toujours possible d'arriver à nos fins, peu importe les embûches rencontrées en chemin!

L'exposition virtuelle est disponible ici : http://www.iaf.inrs.ca/exposition/ fr/armand-frappier

François CARTier 


\section{NOTES}

1. Pour plus d'informations sur l'INRS, voir: http://www.inrs.ca/universite/inrs-bref.

2. Un bon aperçu de la carrière du $D^{r}$ Frappier se trouve ici : http://www.iaf.inrs.ca/iaf/ centre/75-oeuvre-armand-frappier.

3. L'institut est fondé en 1938 sous le nom de l'Institut de microbiologie et d'hygiène de I'Université de Montréal. II est un des premiers à occuper des locaux dans les nouveaux bâtiments sur la montagne de l'Université de Montréal.

4. Ses mémoires sont achevés quelques jours à peine avant son décès, le 17 décembre 1991. Voir FRAPPIER, A. (1992). Un rêve, une lutte: autobiographie. Sillery, Québec: Presses de I'Université du Québec.

5. Le film, intitulé Armand Frappier, est disponible gratuitement sur le site de I'ONF (www.onf.ca).

6. Logiciel que nous avons trouvé depuis et que nous utiliserons éventuellement pour nos archives historiques.

7. Voir https://www.accesstomemory.org/fr/.

8. Voir http://www.ica.org/en/public-resources/standards.

9. Artefactual Systems, qui a développé le logiciel AtoM, offre aussi la possibilité d’héberger le logiciel, et nos données, sur ses propres serveurs.

10. Il s'agissait du Fonds communautaire de commémoration des guerres mondiales.

11. Voir http://www.musee-afrappier.qc.ca.

12. Pour ajouter à sa signification, cette conférence a été donnée dans la bibliothèque du Cégep de Valleyfield, nommée «Bibliothèque Armand-Frappier». 